\title{
MODELING EXTREME VALUES OF PROCESSES OBSERVED AT IRREGULAR TIME STEPS: APPLICATION TO SIGNIFICANT WAVE HEIGHT. SUPPLEMENTARY MATERIAL
}

\author{
By NiCOLAS RAILLARD*
}

Laboratoire de Mathématiques de Bretagne Atlantique, UMR 6205, Université de Brest Laboratoire d'Océanographie Spatiale, IFREMER

Insititut de Recherche Mathématique de Rennes, UMR 6625, Université de Rennes 1

By Pierre Ailliot

Laboratoire de Mathématiques de Bretagne Atlantique, UMR 6205, Université de Brest $\mathrm{AND}$

\section{By JiAnFEng YAO} Department of Statistics \& Actuarial Sciences, The University of Hong Kong

1. Consistency of $\mathbf{M P L}_{1}$ estimator. In this supplementary material, we prove the following theorem.

TheOREM 1.1. Let $\left\{Z_{t}\right\}$ be a Gaussian extreme value process with unit Fréchet margins, as defined in the paper, and dependence parameter $\nu^{*} \in \Theta=\left[\nu_{-}, \nu_{+}\right]$with $0<\nu_{-}<\nu_{+}$. Assume that the process is observed at regular time $\{1 \ldots n\}$, and denote by

$$
L_{n}(\nu)=\prod_{i=1}^{n-1} \frac{\partial^{2} F_{Z}}{\partial z_{1} \partial z_{2}}\left(Z_{i}, Z_{i+1} ; \nu\right)
$$

the pairwise likelihood function, with $F_{z}$ defined by

$$
F_{Z}\left(z_{t_{1}}, z_{t_{2}} ; \nu\right)=\mathbb{P}\left(Z_{t_{1}} \leq z_{t_{1}}, Z_{t_{2}} \leq z_{t_{2}}\right)=\exp \left[-V\left(z_{t_{1}}, z_{t_{2}} ; \nu\right)\right]
$$

and

$$
V\left(z_{t_{1}}, z_{t_{2}} ; \nu\right)=\frac{1}{z_{t_{1}}} \Phi\left(\frac{a}{2}+\frac{1}{a} \log \frac{z_{t_{2}}}{z_{t_{1}}}\right)+\frac{1}{z_{t_{2}}} \Phi\left(\frac{a}{2}+\frac{1}{a} \log \frac{z_{t_{1}}}{z_{t_{2}}}\right) .
$$

We define $\hat{\nu}_{n}=\operatorname{argmax} L_{n}(\nu)$ as the corresponding estimator of $\nu^{*}$. Then, $\hat{\nu}_{n}$ is a strongly consistent estimator of $\nu^{*}$, i.e.,

$$
\lim _{n \rightarrow \infty} \hat{\nu}_{n}=\nu^{*} \text { a.s. }
$$

\footnotetext{
${ }^{*}$ Corresponding Author (E-mail: nicolas.raillard@gmail.com)
} 
A Gaussian extremal process $\left\{Z_{t}\right\}$ is a moving maxima process as defined in Stoev (2008). Using the results given in that paper, we deduce that $\left\{Z_{t}\right\}$ is a stationary unit Fréchet process, continuous in probability, mixing, and hence ergodic. It allows us to use the following theorem, which is a straightforward generalization of Theorem 1.12 in Pfanzagl (1969):

THEOREM 1.2. Let $\left\{Z_{i}\right\}_{i=1, \ldots, n}$ be a stationary and ergodic process, whose distribution depends on a parameter $\nu^{*} \in \Theta$, where $\Theta$ is a compact subset of $\mathbb{R}$, and let $Q_{n}$ be a contrast function defined as

$$
Q_{n}(\nu)=\frac{1}{n} \sum_{i=1}^{n-1} f\left(Z_{i}, Z_{i+1} ; \nu\right)
$$

where $f$ is a measurable function with real values and continuous in $\nu$. Suppose that:

(a) $\mathbb{E} \inf _{\nu \in \Theta} f\left(Z_{1}, Z_{2} ; \nu\right)>-\infty$;

(b) $\nu \mapsto \mathbb{E} f\left(Z_{1}, Z_{2} ; \nu\right)$ has a unique finite minimum at $\nu^{*}$.

Then, the minimum contrast estimator $\hat{\nu}_{n}=\operatorname{argmin}_{\nu \in \Theta} Q_{n}(\nu)$ is strongly consistent:

$$
\lim _{n \rightarrow \infty} \hat{\nu}_{n}=\nu^{*} \text { a.s. }
$$

We use this theorem with

$$
f\left(Z_{1}, Z_{2} ; \nu\right)=-\log p\left(Z_{1}, Z_{2} ; \nu\right)
$$

where $p\left(z_{1}, z_{2} ; \nu\right)$ denotes the joint pdf of $\left(Z_{1}, Z_{2}\right), \Theta=\left(\nu_{-}, \nu_{+}\right)$with $0<\nu_{-}<\nu_{+}$. An explicit expression for $f$ is given in Section 1.1. Properties (1) and (2) of Theorem 1.2 are verified in Sections 1.2 and 1.3, respectively.

1.1. Expression of the contrast. We have

$$
p\left(Z_{1}, Z_{2} ; \nu\right)=\frac{\partial^{2}}{\partial z_{1} \partial z_{2}} F_{Z}\left(z_{1}, z_{2} ; \nu\right)
$$

with $F_{Z}\left(z_{1}, z_{2} ; \nu\right)=\exp \left(\left(-V\left(z_{1}, z_{2} ; \nu\right)\right)\right.$ and $V$ defined in $(1)$ and thus

$$
f\left(Z_{1}, Z_{2} ; \nu\right)=V\left(z_{1}, z_{2} ; \nu\right)-\log \left(\frac{\partial V}{\partial z_{1}}\left(z_{1}, z_{2}\right) \frac{\partial V}{\partial z_{2}}\left(z_{1}, z_{2}\right)+\frac{\partial^{2} V}{\partial z_{1} \partial z_{2}}\left(z_{1}, z_{2}\right)\right) .
$$


The function $V$ and its derivatives satisfy

$$
\begin{aligned}
V\left(z_{1}, z_{2} ; \nu\right) & =\frac{\Phi\left(a / 2+1 / a \log \frac{z_{2}}{z_{1}}\right)}{z_{1}}+\frac{\Phi\left(a / 2+1 / a \log \frac{z_{1}}{z_{2}}\right)}{z_{2}}=\frac{\Phi(w)}{z_{1}}+\frac{\Phi(v)}{z_{2}}, \\
\frac{\partial V}{\partial z_{1}}\left(z_{1}, z_{2} ; \nu\right) & =-\frac{\Phi(w)}{z_{1}^{2}}-\frac{\varphi(w)}{a z_{1}^{2}}+\frac{\varphi(v)}{a z_{1} z_{2}}, \\
\frac{\partial V}{\partial z_{2}}\left(z_{1}, z_{2} ; \nu\right) & =-\frac{\Phi(v)}{z_{2}^{2}}-\frac{\varphi(v)}{a z_{2}^{2}}+\frac{\varphi(w)}{a z_{1} z_{2}}, \\
\frac{\partial^{2} V}{\partial z_{1} \partial z_{2}}\left(z_{1}, z_{2} ; \nu\right) & =-\frac{v \varphi(w)}{a^{2} z_{1}^{2} z_{2}}-\frac{w \varphi(v)}{a^{2} z_{1} z_{2}^{2}}
\end{aligned}
$$

with

$$
\begin{aligned}
w & =\frac{a}{2}+\frac{1}{a} \log \frac{z_{2}}{z_{1}}, \\
v & =a-w=\frac{a}{2}+\frac{1}{a} \log \frac{z_{1}}{z_{2}}, \\
\varphi(w) & =\frac{1}{\sqrt{2 \pi}} e^{-a^{2} / 8} e^{-\frac{\log ^{2}\left(z_{1} / z_{2}\right)}{2 a^{2}}} \sqrt{\frac{z_{1}}{z_{2}}} \\
\varphi(v) & =\frac{1}{\sqrt{2 \pi}} e^{-a^{2} / 8} e^{-\frac{\log ^{2}\left(z_{1} / z_{2}\right)}{2 a^{2}}} \sqrt{\frac{z_{2}}{z_{1}}} .
\end{aligned}
$$

Taking into account that $\frac{\varphi(w)}{a z_{1}^{2}}-\frac{\varphi(v)}{a z_{1} z_{2}}=0$, the following simplified expressions for the derivatives of $V$ are obtained:

$$
\begin{gathered}
\frac{\partial V}{\partial z_{1}}\left(z_{1}, z_{2}\right)=-\frac{\Phi(w)}{z_{1}^{2}}, \\
\frac{\partial V}{\partial z_{2}}\left(z_{1}, z_{2}\right)=-\frac{\Phi(v)}{z_{2}^{2}}, \\
\frac{\partial^{2} V}{\partial z_{1} \partial z_{2}}\left(z_{1}, z_{2}\right)=-\frac{\varphi(w)}{a z_{1}^{2} z_{2}}=-\frac{\varphi(v)}{a z_{2}^{2} z_{1}}=\frac{e^{-a^{2} / 8} \exp \left(\frac{\log ^{2} \frac{z_{2}}{z_{1}}}{2 a^{2}}\right)}{\left(z_{1} z_{2}\right)^{3 / 2}} .
\end{gathered}
$$

Finally, we deduce that

$$
f\left(z_{1}, z_{2} ; \nu\right)=V\left(z_{1}, z_{2} ; \nu\right)-\log \left[\frac{\Phi(w) \Phi(v)}{a^{2} z_{1} z_{2}}+\frac{\varphi(w)}{a z_{1}^{2} z_{2}}\right]
$$

1.2. Lower bound (a). We have

$$
\inf _{\nu}\left\{f\left(z_{1}, z_{2} ; \nu\right)\right\} \geq \inf _{\nu}\left\{V\left(z_{1}, z_{2} ; \nu\right)\right\}+\inf _{\nu}\left\{-\log \left[\frac{\Phi(w) \Phi(v)}{a^{2} z_{1} z_{2}}+\frac{\varphi(w)}{a z_{1}^{2} z_{2}}\right]\right\}
$$

and each term on the right-hand side of this expression is treated separately below. 
- $\operatorname{Term} V\left(z_{1}, z_{2} ; \nu\right)$.

$V$ can be bounded using the Fréchet bound (Fréchet (1951)),

$P\left(Z_{1} \leq z_{1}\right)+P\left(Z_{2} \leq z_{2}\right)-1 \leq \mathbb{P}\left(Z_{1} \leq z_{1}, Z_{2} \leq z_{2}\right) \leq \min \left\{P\left(Z_{1} \leq z_{1}\right), P\left(Z_{2} \leq z_{2}\right)\right)$,

which implies that

$$
\min \left(-\frac{1}{z_{1}},-\frac{1}{z_{2}}\right) \leq V\left(z_{1}, z_{2} ; \nu\right) \leq \exp \left(-\frac{1}{z_{1}}\right)+\exp \left(\frac{1}{z_{2}}\right)
$$

and thus

$$
\inf _{\nu}\left\{V\left(z_{1}, z_{2} ; \nu\right)\right\} \geq \min \left(-\frac{1}{z_{1}},-\frac{1}{z_{2}}\right) .
$$

The right-hand term of the last expression has a finite expectation since $\frac{1}{Z_{1}}$ and $\frac{1}{Z_{2}}$ have unit exponential distributions.

- Term $-\log \left[\frac{\Phi(w) \Phi(v)}{a^{2} z_{1} z_{2}}+\frac{\varphi(w)}{a z_{1}^{2} z_{2}}\right]$.

We have

$$
\begin{aligned}
-\log \left[\frac{\Phi(w) \Phi(v)}{z_{1} z_{2}} \nu^{2}+\frac{\varphi(w)}{z_{1}^{2} z_{2}} \nu\right] & \geq 1-\frac{\Phi(w) \Phi(v)}{z_{1} z_{2}} \nu^{2}+\frac{\varphi(w)}{z_{1}^{2} z_{2}} \nu \\
& \geq 1-\frac{\nu^{2}}{z_{1} z_{2}} \\
& \geq 1-\frac{\nu_{+}^{2}}{z_{1} z_{2}}
\end{aligned}
$$

and Cauchy's inequality implies that the right-hand term of the last expression has a finite expectation since

$$
\mathbb{E}\left[\frac{1}{Z_{1} Z_{2}}\right] \leq \sqrt{\mathbb{E}\left[1 / Z_{1}^{2}\right] \mathbb{E}\left[1 / Z_{2}^{2}\right]}=1
$$

1.3. Identifiability (b). We must now prove that $\nu \mapsto \mathbb{E} f\left(Z_{1}, Z_{2} ; \nu\right)$ has a unique finite minimum at $\nu^{*}$ on $\Theta=\left[\nu_{-} \nu_{+}\right]$. To this end we denote by $P_{\nu}$ the distribution with density function $p\left(z_{1}, z_{2} ; \nu\right)$. Then,

$$
\mathbb{E}_{\nu^{*}}\left[-\log \frac{p\left(Z_{1}, Z_{2} ; \nu\right)}{p\left(Z_{1}, Z_{2} ; \nu^{*}\right)}\right]=K\left(P_{\nu^{*}}, P_{\nu}\right)
$$

is the Kullback-Leibler divergence between $P_{\nu^{*}}$ and $P_{\nu}$. We have $K \geq 0$ and $K=0$ iff $P_{\nu}=P_{\nu^{*}}$. As the density functions are positive and continuous in $\left(z_{1}, z_{2}\right)$, this is equivalent 
to $p\left(z_{1}, z_{2} ; \nu^{*}\right)=p\left(z_{1}, z_{2} ; \nu\right)$ for all $\left(z_{1}, z_{2}\right)$. In particular, $K=0$ implies that for all $z_{1}=$ $z_{2}=z>0$, we have

$\exp \left[-\frac{2}{z} \Phi\left(\frac{1}{2 \nu}\right)\right]\left[\frac{\Phi\left(\frac{1}{2 \nu}\right)^{2}}{z^{2}} \nu^{2}+\frac{\varphi\left(\frac{1}{2 \nu}\right)}{z^{3}} \nu\right]=\exp \left[-\frac{2}{z} \Phi\left(\frac{1}{2 \nu^{*}}\right)\right]\left[\frac{\Phi\left(\frac{1}{2 \nu^{*}}\right)^{2}}{z^{2}} \nu^{* 2}+\frac{\varphi\left(\frac{1}{2 \nu^{*}}\right)}{z^{3}} \nu^{*}\right]$

Letting $z \rightarrow 0$ while $z>0$ we see that the exponents in the exponential function must be equal, i.e.,

$$
\Phi\left(\frac{1}{\nu}\right)=\Phi\left(\frac{1}{\nu^{*}}\right)
$$

Hence, $\nu=\nu^{*}$. The proof is complete.

\section{References.}

FrÉchet, M. (1951). Sur les tableaux de corrélation dont les marges sont données. Annales de l'Université de Lyon 14 53-77. MR0049518 (14,189c)

Pfanzagl, J. (1969). On the measurability and consistency of minimum contrast estimates. Metrika 14 249-272.

Stoev, S. A. (2008). On the ergodicity and mixing of max-stable processes. Stochastic Processes and their Applications 118 1679-1705. MR2442375 (2009e:60116) 